[Aus dem hygienischen Institut zu Berlin.]

\title{
Mikroskopische Untersuchungen des Darminhaltes von an Cholera asiatica verstorbenen Indiern.

\author{
Von
}

Stabsarzt Dr. Weisser und Dr. med. Georg Frank, Assistenten am hygienischen Institut.

Am ersten Sitzungstage der zweiten Conferenz zur Erörterung der Cholerafrage machte Geheimrath Koch im Anschlusse an seinen Bericht über die weiteren Ergebnisse der Choleraforschung seit der vorhergegangenen Conferenz Mittheilung über Untersuchungen von Deckglaspräparaten des Darminhaltes von an Cholera verstorbenen Indiern, die ihm durch die Güte des Hrn. Dr. Dissent, Arzt am Sealdah Hospital zu Calcutta, zugegangen waren.

$\mathrm{Da}$ in den Berichten über die Choleraconferenz dieser Untersuchung nur kurz Erwähnung gethan ist, und da im Laufe des rergangenen Jahres noch einige neue Fälle hinzugekommen sind, so berichten wir hier über diese Untersuchungen etwas ausführlicher, weil wir glauben, dass sie die Beobachtungen derjenigen Forscher nach Koch, die, wie van Ermenghem, Watson Cheyne, Ceci, Nicati u. Rietsch, Babes, Pfeiffer u. s. w., die Anwesenheit der Cholerabacterien bei Cholera constatirten, ergänzen und als Erweis dafür dienen können, dass die mikroskopische Untersuchung allein in der Mehrzahl der Fälle genügen kann, die Diagnose auf Cholera asiatica zu stellen.

Diese Deckglaspräparate waren in der Weise angefertigt, dass die ganze Oberfläche des Deckglases ausgenutzt war. Nach der mikroskopischen Untersuchung scheint die Annahme berechtigt, als ob zum Ausstriche nicht immer die charakteristischen Schleimflocken des Inhaltes des Choleradarmes gewählt worden seien, in denen die Kommabacillen in besonders 
dichter Menge zu sitzen pflegen, sondern die mehr wässerigen und an Kommabacillen ärmeren Bestandtheile des Darminhaltes. Es zeigte sich nämlich in vielen Präparaten eine viel geringere Menge von Schleim und Epithel als wir in den von Geheimrath $K_{0 c h}$ angefertigten Prïparaten gesehen hatten. Wenn auch durch diesen Cmstand Schwierigkeiten, die bei einem vorschriftsmässigen Ausstreichen hätten vermieden werden können, dem mikroskopischen Nachweise der Fíommabacillen entgegentraten, so gelang es duch durch genaue rergleichende Durchsicht der Praeparate, die meist in der Zahl von fünf oder sechs ron verschiedenen Stellen des Darmes entnommen in jedem einzelnen Falle vorlagen, doch auch in jenen Fällen, in denen die Kommabacillen nur sehr spärlich und zerstreut vorkamen, ihre Anwesenheit in dem einen oder anderen der Präparate zu constatiren.

Gleichzeitig hatte Dr. Dissent jedem Falle - mit Ausnahme ron zweien - eine kurze Notiz über Namen, Alter, Stand des Erkrankten, Beginn, Verlauf und Ende der Krankheit beigegeben.

Von den übersandten Fällen - nur wenige Deckgläser waren auf dem Transporte verdorben und zur Tntersuchung nicht mehr geeignet, so dass die betreffenden Fälle hier ganz ausgeschlossen bleiben - sind 90 mikroskopisch untersucht worden. Die Deckglaspräparate, die in Calcutta einfach an der Luft getrocknet waren, wurden zu dem Zwecke der Untersuchung direct mit den geeigneten Anilinfarben, meistens Fuchsinlösung, gefärbt, da bei der langen Zeit, die nach dem Bestreichen der Deckgläser bis zur mikroskopischen Untersuchung vergangen war, die Abstriche an den Deckgläsern so fest angetrocknet waren, dass das gewöhnlich geübte Fixiren derselben durch Durchziehen durch die Flamme nicht mehr nöthig war.

Bei der mikroskopischen Untersuchung wurde nicht allein auf das Vorkommen der Kommabacillen überhaupt geachtet, sondern auch die ungeführe Menge derselben in den constatirten Fällen durch die Bezeichnungen: „wenig oder spärlich, reichlich oder viel" „Reincultur oder fast Reincultur" annähernd zu bestimmen gesucht, und sind die Fälle erster Rubrik mit einem, die zweiter mit zwei, die dritter mit drei Kreuzen in der Tabelle versehen worden. (Siehe S. 382-389).

Von den 90 untersuchten Fällen - 89 Obductionen 1 Kranker - wurden die Kommabacillen durch die mikroskopische Untersuchung 83 mal constatirt; 7 mal wurden sie vermisst. Von diesen sieben Fällen waren in zwei die Präparate in Folge von Schimmelpilzwucherungen rerdorben. In den fünf übrigen Fällen fanden sich in den Präparaten sehr riele verschieden geformte Bacterien und reichlich Blut; von diesen fünfen ist in der beigegebenen Krankengeschichte einer als im Reactionsstadium versturben 
bezeichnet. Aber auch in den vier übrigen Fällen fand sich den Präparaten Blut so reichlich beigemischt, dass man wohl annehmen darf, dass auch diese Fällen angehörten, die im Reactionsstadium gestorben sind. Vielleicht könnte es sich auch in dem einen oder anderen dieser Fälle nicht um Cholera, sondern um jenes eigenthümliche perniciöse Fieber Indiens gehandelt haben, das unter Symptomen rerläuft, welche denen der Cholera asiatica sehr ähnlich sind, und welches ein so wenig charakteristisches pathologisch-anatomisches Bild hinterlässt, dass selbst dem mit den tropischen Krankheiten rertrauten Arzte die Differentialdiagnose sehr schwer, sogar unmöglich werden kann.

Von den 83 Fällen, in denen die Kommabacillen gefunden wurden, sind

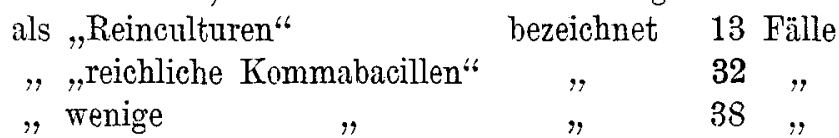

In den 13 Fällen „Reineultur“" wurde 8 mal das Blut vermisst; 5 mal wurde nur wenig constatirt.

In den 32 Fällen, ,reichliche Kommabacillen" wurde kein Blut gefunden bei 15, wenig bei 13, riel bei 3 ; in einem Falle war, weil das Präparat durch Schimmelpilzwucherungen beschädigt, kein sicherer Entscheid zu geben.

In den 38 Fällen ,wenige Kommabacillen" wurde kein Blut constatirt bei 6 , wenig Blut bei 21, sehr viel Blut bei 9 Fällen; unbestimmbar aus dem oben angeführten Grunde war es in zwei Fällen.

Von den 83 Fällen, bei denen die Kommabacillen constatirt wurden, starben

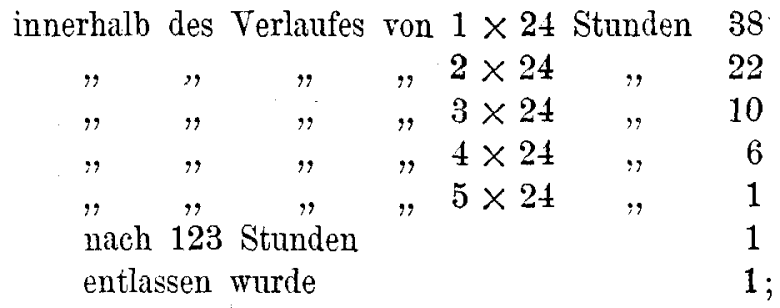

eine Bestimmung über die Krankheitsdauer konnte aus den beigegebenen Notizen nicht gezogen werden in 4 Fällen.

Die Untersuchungen berechtigen uns zu folgenden Schlüssen:

Zuerst möchten wir, Klein's Behauptung gegenüber, dass bei ganz rapidem Verlaufe der Cholera die Kommabacillen rermisst würden, constatiren, dass in keinem der Fälle, die weniger als zwölf Stunden gedauert haben, die Kommabacillen fehlten, dass sie rielmehr in den meisten fast in Reincuitur vorkamen. 


\begin{tabular}{|c|c|c|c|c|c|}
\hline Nr. & Name & Geschlecht & $\begin{array}{l}\text { Jahre } \\
\text { Alter }\end{array}$ & Wann erkrankt & Wann gestorben \\
\hline 1 & $\begin{array}{l}\text { Krankengeschichte } \\
\text { fehlt. }\end{array}$ & & & & \\
\hline 2 . & Bipol (Hindu) & männlich . & 40 & 14. Febr. 85 früh Morg. & 15. Febr. $18859^{\text {h }}$ a. m. \\
\hline 3 & Banka & $"$ & 8 & 17. , $5^{\mathrm{h}}$ a. m. & 17. , $10^{\mathrm{h}}$ p. m. \\
\hline 4 & Mohnu Call (Hindu) & $"$ & 34 & 21. , $3^{\text {h }}$ p. m. & 22. , $6^{\text {h }}$ a. $\mathrm{m}$. \\
\hline 5 & Streiram (Hindu) & $"$, & 34 & 21. , $9^{\mathrm{h}}$ p. m. & 22. , $12^{\text {h }}$ Mittern. \\
\hline 6 & Maha hunda (Hindu) & " & $3 \tilde{5}$ & $22 \quad, \quad 5^{\mathrm{h}}$ a. $\mathrm{m}$. & 22. , $\quad 4^{\mathrm{h}}$ p.m. \\
\hline 7 & Jajeshari (Hindu) & weiblich & $?$ & 26. , $\quad 3^{\mathrm{k}}$ a. $\mathrm{m}$ & 27. , $6^{\text {he }}$ a. $m$. \\
\hline 8 & $\begin{array}{l}\text { Ranglio Janger } \\
\text { (Hindu). }\end{array}$ & snännlich & $?$ & 27. , $12^{4} 30$ a. m. & $\begin{array}{ll} & \text { entlassen } \\
\text { am 4. März 18sō. }\end{array}$ \\
\hline 9 & Puddo (Hindu) & weiblich & 50 & 1. März $3^{\mathrm{n}}$ a. m. & 2. März $6^{\text {h }}$ a. m. \\
\hline 10 & Soorut (Hindu) & männlich & 20 & 1. ", in der Nacht & 4. , $6^{\mathrm{L}}$ a. m. \\
\hline 11 & Surja & - & - & - & 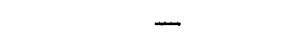 \\
\hline 12 & Indu (Hindu) & männlich & 50 & 3. , $11^{12}$ a. m. & 4.,$\quad 5^{\mathrm{h}}$ a. $\mathrm{m}$. \\
\hline 13 & Siboo (Hindu) & , & 40 & 4. , $\quad 6^{\mathrm{h}}$ a. m. & 9. , $\quad 1^{\text {he }}$ a. $\mathrm{m}$ \\
\hline 14 & Ihagia (Hindu) & weiblich & - & 5. , $11^{\mathrm{h}}$ a. $\mathrm{m}$. & 8. , $\quad 7^{\text {h }}$ p. $\mathrm{m}$. \\
\hline 15 & Bhatoo (Hindu) & männlich & 35 & $\begin{array}{c}\text { 8. ", während der } \\
\text { Nacht }\end{array}$ & 9. , $11^{\mathrm{b}}$ a. $\mathrm{m}$. \\
\hline 16 & Lukti Narain & , & $4 \check{5}$ & 8. , & 11. , $12^{\mathrm{h}}$ a. $1 \mathrm{~m}$. \\
\hline 17 & Gopal (Hindu) & , & Jüngl. & 10. , $6^{\mathrm{L}}$ p. m. & 12. , $6^{\mathrm{b}}$ a. $\mathrm{m}$. \\
\hline 18 & Sliputi (Hindu) & " & $"$ & 10. , $12^{\mathrm{h}}$ a. $\mathrm{n}$. & 12. , , $4^{h}$ p. 3. \\
\hline 19 & $\underset{\text { daner) }}{\text { Ramjanaky (Muhame- }}$ & $"$ & 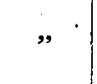 & 12. , $11^{\text {h }}$ a. $m$. & 13. , $10^{\mathrm{h}} \mathrm{p} \cdot \mathrm{m}$. \\
\hline 20 & Heera (Hindu) & weiblich & , & 12. , Nachts & 14. , $1^{\text {lh }}$ a. In. \\
\hline 21 & Som nath (Hindu) & männlich & 35 & 12. , " & 13. , $12^{\mathrm{h}}$ a. $\mathrm{m}$. \\
\hline 22 & Nautuni (Hindu) & 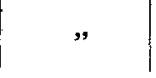 & Jüngl. & 12. , & 14. , $\quad 2^{\text {h }}$ p. $1 \mathrm{~m}$. \\
\hline
\end{tabular}




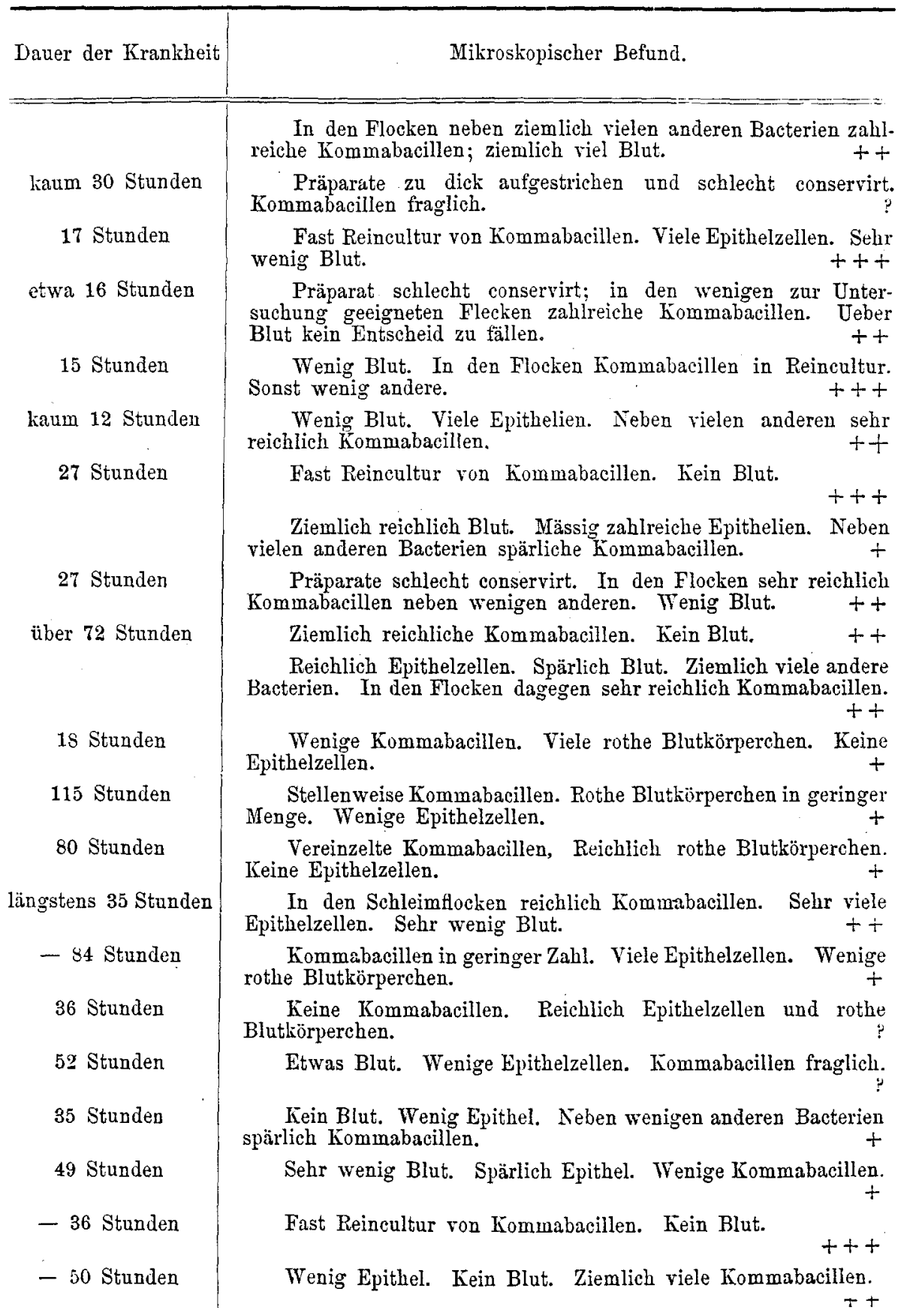




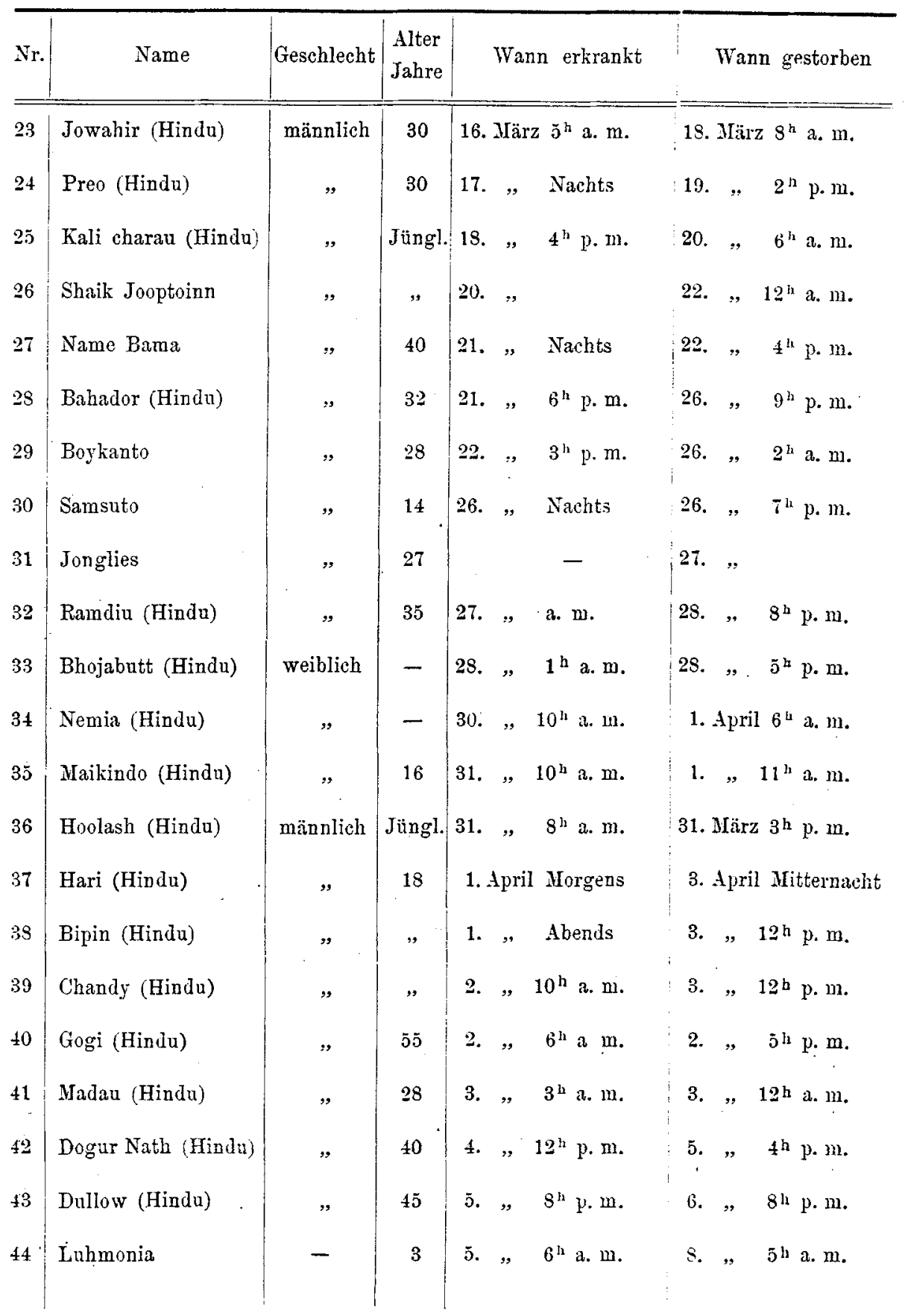




\begin{tabular}{|c|c|}
\hline Dauer der Krankheit & Mikroskopischer Befund. \\
\hline 51 Stunden & $\begin{array}{l}\text { Wenig Epithel. Kein Blut. In den Flocken zahlreiche Komma- } \\
\text { bacillen. }\end{array}$ \\
\hline - 50 Stunden & $\begin{array}{l}\text { Sehr viele rothe Blutkörperchen. Wenig Epithel. Neben sehr } \\
\text { vielen anderen Bacterien spärlich Kommabacillen. }\end{array}$ \\
\hline 38 Stunden & $\begin{array}{l}\text { In einigen Präparaten reichlich rothe Blutkörperchen, in an- } \\
\text { dern keine. Wenig Epithel. Ziemlich zahlr. Kommabacillen. + }+\end{array}$ \\
\hline - 60 Stunden & $\begin{array}{l}\text { Wenige Kommabacillen. Sehr viele rothe BIutkörperchen. } \\
\text { Keine Epithelzellen. }\end{array}$ \\
\hline-40 Stunden & $\begin{array}{l}\text { Reichlich Epithel. Kein Blut. In den Flocken Kommabacillen } \\
\text { in reicher Anzahl. }\end{array}$ \\
\hline 123 Stunden & $\begin{array}{l}\text { Sehr wenige Kommabacillen. Wenige Epithelzellen. Verein- } \\
\text { zelte rothe Blutkörperchen. }\end{array}$ \\
\hline 83 Stunden & $\begin{array}{l}\text { Wenige Kommabacillen. Wenige rothe Blutkörperchen. Ver- } \\
\text { einzelte Epithelzellen. }\end{array}$ \\
\hline - 19 Stunden & $\begin{array}{l}\text { Wenige Kommabacillen. Sehr viele Epithelzellen. Keine rothen } \\
\text { Blutkörperchen. }\end{array}$ \\
\hline $4^{\text {h }}$ nach der Aufnahme & $\begin{array}{ll}\text { Kommabacillen in Schleimflocken in grösserer Menge. Sehr } \\
\text { viele Epithelzellen. Keine rothen Blutkörperchen. }\end{array}$ \\
\hline - 32 Stunden & $\begin{array}{l}\text { Spärliche Kommabacillen. Keine Epithelzellen. Wenige rothe } \\
\text { Blutkörperchen. }\end{array}$ \\
\hline 16 Stunden & $\begin{array}{l}\text { Keine Kommabacillen. Keine Epithelzellen. Wenige rothe } \\
\text { Blutkörperchen. }\end{array}$ \\
\hline 44 Stunden & $\begin{array}{l}\text { Vereinzelte Kommabacillen. Wenige zerfallene rothe Blut- } \\
\text { körperchen, sowie Epithelzellen. }\end{array}$ \\
\hline 25 St. n. d. Aufnahme & $\begin{array}{lrr}\text { Wenige Kommabacillen. } & \text { Wenige Epithelzellen. } & \text { Reichlich } \\
\text { rothe Blutkörperchen. } & +\end{array}$ \\
\hline 7 Stunden & $\begin{array}{l}\text { Reinoultur von Kommabacillen. Sehr viele Epithelzellen. Sehr } \\
\text { wenig Blut. } \\
+++\end{array}$ \\
\hline - 72 Stunden & $\begin{array}{l}\text { Sehr viele rothe Blutkörperchen. Verschiedene Bacterien in } \\
\text { grosser Zahl. Kommabacillen fraglich. }\end{array}$ \\
\hline - 54 Stunden & $\begin{array}{l}\text { Viele rothe Blutkörperchen. Sehr viele verschiedene Bacterien. } \\
\text { Kommabacillen fraglich. }\end{array}$ \\
\hline 38 Stunden & $\begin{array}{l}\text { Sehr viele verschiedene Bacterien. Viele rothe Blutkörperchen. } \\
\text { Wenige Kommabacillen. }\end{array}$ \\
\hline - 11 Stunden & $\begin{array}{lcc}\text { Reichlich Kommabacillen. Keine Epithelzellen. Sehr viele } \\
++ \\
\text { rothe Blutkörperchen. }\end{array}$ \\
\hline 9 Stunden & $\begin{array}{l}\text { Wenige Kommabacillen. Keine Epithelzellen. Wenige rothe } \\
\text { Blutkörperchen. }\end{array}$ \\
\hline 16 Stunden & $\begin{array}{l}\text { In einem Präparat in den Flocken Reincultur von Komma- } \\
\text { bacillen, in den anderen spärliche neben Blut und Epithel. }+++\end{array}$ \\
\hline 24 Stunden & $\begin{array}{l}\text { Rothe Blutkörperchen. Spirochaeten. Viele andere Bacterien, } \\
\text { darunter charakteristische Kommabacillen in geringer Zahl. + }\end{array}$ \\
\hline 71 Stunden & $\begin{array}{l}\text { Wenig Blut. Spärlich Epithel. Kommabacillen in geringer } \\
\text { Zahl in Flocken. }\end{array}$ \\
\hline
\end{tabular}




\begin{tabular}{|c|c|c|c|c|c|c|c|c|c|c|c|}
\hline Nr. & Name & Geschlecht & $\begin{array}{l}\text { Alter } \\
\text { Jahre }\end{array}$ & \multicolumn{4}{|c|}{ Wann erkrankt } & \multicolumn{4}{|c|}{ Wann gestorben } \\
\hline 45 & Ihumon (Hindu) & männlich & 36 & & April & $110^{1}$ & h a. $\mathrm{m}$. & & Apri & $1^{\mathrm{h}}$ & a. m. \\
\hline 46 & Kuknowu (Hindu) & " & - & 8. & " & a. & m. & 8. & , & $1^{\mathrm{h}}$ & p. m. \\
\hline 47 & Gungaram (Hindu) & " & 60 & 8. & , & p. & $\mathrm{m}$. & 10. & , & $4^{b}$ & a. $\mathrm{m}$. \\
\hline 48 & Bidhu Mookhy(Hindu) & weiblich & 70 & 8. & $"$ & $\mathrm{Nac}$ & chmittags & 9. & , & $6 \mathrm{~h}$ & p. m. \\
\hline 49 & Nela Sing (Hindu) & männlich & 45 & 8. & , & $\mathrm{Na}$ & $\operatorname{chts}$ & 9. & ", & $3^{\mathrm{h}}$ & p. m. \\
\hline 50 & Durbally (Hindu) & , & 35 & 10 . & , & & a. $\mathrm{m}$. & 11. & " & $6^{\mathrm{h}}$ & a. $\mathrm{m}$. \\
\hline 51 & Akla (Hindu) & weiblich & - . & 12. & , & $4^{b}$ & a. $\mathrm{m}$. & 12. & , & $1^{\mathrm{h}}$ & p. m. \\
\hline 52 & Durga Claru (Hindu) & männlich & 40 & 12. & ", & & & $1 \overline{5}$. & , & $10^{\mathrm{h}}$ & a. $\mathrm{m}$. \\
\hline 53 & Parma (Hindu) & $"$ & 35 & 15 . & " & $\mathbf{2}^{\mathbf{h}}$ & a. $\mathrm{m}$. & 15. & $"$ & $\mathbf{2 h}^{\mathbf{h}}$ & p. $\mathrm{m}$. \\
\hline 54 & Munsary (Hindu) & , & 40 & 14. & , & & a. $\mathrm{m}$. & 17. & " & $4^{h}$ & p. m. \\
\hline 55 & $\begin{array}{l}\text { Menajoddi (Muhame- } \\
\text { daner) }\end{array}$ & " & 30 & 16. & , & & & 17. & , & $4^{\mathrm{h}}$ & p. m. \\
\hline 56 & Akhoy (Hindu) & , & 28 & 16 . & , & $12 \mathrm{~h}$ & a. $\mathrm{m}$. & 17. & " & $6^{\mathrm{h}}$ & p. $\mathbf{m}$. \\
\hline 57 & Nitai & - & 26 & 19. & , & $4^{h}$ & a. $\mathrm{m}$. & 19. & , & $2^{h}$ & p. m. \\
\hline 58 & Konodos (Hindu) & männlich & 30 & 17. & , & & & 19. & " & $11^{\mathrm{h}}$ & a. $\mathrm{m}$. \\
\hline 59 & Kurum (Mubamed.) & " & 30 & 29. & " & & a. $\mathrm{m}$. & 30. & , & $6^{\mathrm{h}}$ & a. $\mathrm{m}$. \\
\hline 60 & Gopiram (Hindu) & ", & 36 & 29. & " & $12^{\mathrm{b}}$ & a. $\mathrm{m}$. & 30. & , & $6^{\mathrm{h}}$ & a. $\mathrm{m}$. \\
\hline 61 & Gungadun (Hindu) & " & 25 & 30. & " & & a. $\mathrm{m}$. & 2. & Mai & $5^{\mathrm{h}}$ & p. $m$. \\
\hline 62 & Ojhala & weiblich & 20 & 6. & Mai & & a. $\mathrm{m}$. & 7. & , & $5 \mathrm{~h}$ & a. m. \\
\hline 63 & Bhola Nath (Hindu) & männlieh & 30 & 5. & , & $6 \mathrm{~h}$ & a. $\mathrm{m}$. & 7. & , & $5^{\mathbf{h}}$ & a. $\mathrm{m}$. \\
\hline 64 & Bhikock (Hindu) & weiblich & 27 & 9. & " & & a. $\mathrm{m}$. & 10. & , & $5^{b}$ & p. m. \\
\hline 65 & Hari Bhiwa & " & 42 & 8. & , & & a. $\mathrm{m}$. & 10. & , & $6 \mathrm{~h}$ & a. $\mathrm{m}$. \\
\hline 66 & Mathai (Hindu) & männlich & 40 & 8. & , & & p. m. & 10 . & , & $7 \mathrm{~h}$ & a. $\mathrm{n}$. \\
\hline
\end{tabular}




\begin{tabular}{|c|c|}
\hline Dauer der Krankheit & Mikroskopischer Befund. \\
\hline 15 Stunden & $\begin{array}{l}\text { Kein Blut. Wenig Epithel. In den rielen Flocken fast Rein } \\
\text { cultur von Kommabacillen. }\end{array}$ \\
\hline - 20 Stunden & $\begin{array}{r}\text { Kein Blut. Viel Epithel. Fast Reincultur von Kommabacillen } \\
+++\end{array}$ \\
\hline - 40 Stunden & Etwas Blut. Wenig Epithel. Spärlich Kommabacillen. + \\
\hline 26 Stunden & $\begin{array}{l}\text { Wenig Blut. ziemlich reichlich Epithel. Spärlich Komma- } \\
\text { bacillen. }\end{array}$ \\
\hline 20 Stunden & Kein Blut. Vereinzelte Kommabacillen. + \\
\hline 24 Stunden & $\begin{array}{l}\text { Kein Blut. Wenig Epithel. In den Flocken ziemlich viele } \\
++ \\
\text { Kommabacillen. }\end{array}$ \\
\hline 9 Stunden & $\begin{array}{l}\text { Reichliche Kommabacillen, besonders in Schleimflocken. Reich } \\
\text { lich wohl erbaltene Epithelzellen. Vereinzelte rothe Blutkörperchen. } \\
++\end{array}$ \\
\hline - 58 Stunden & $\begin{array}{l}\text { Wenige Kommabacillen. Keine Epithelzellen. Wenige rothe } \\
\text { Blutkörperchen. }\end{array}$ \\
\hline 12 Stunden & $\begin{array}{l}\text { Reincultur von Kommabacillen. Viele wohl erhaltene Epithel } \\
\text { zellen. Keine rothen Blutkörperchen. } \\
+++\end{array}$ \\
\hline - 88 Ștunden & $\begin{array}{l}\text { Reichliche Kommabacillen. Viele Epithelzellen. Keine rothen } \\
\text { Blutkörperchen. }\end{array}$ \\
\hline - 10 Stunden & $\begin{array}{l}\text { Vereinzelte Kommabacillen. Keine Epithelzellen. Sehr wenige } \\
\text { rothe Blatkörperchen. }\end{array}$ \\
\hline 30 Stunden & $\begin{array}{l}\text { Häufige Kommabacillen in Zügen. Fereinzelte Epithelzellen. } \\
\text { Wenige rothe Blutkörperchen. }\end{array}$ \\
\hline 40 Stunden & $\begin{array}{l}\text { Mässige Menge Kommabacillen, aber fast in Reincultar. Viele } \\
\text { Epithelzellen. Wenige rothe Blutkörperchen. }\end{array}$ \\
\hline - 59 Stunden & $\begin{array}{l}\text { Wenige Kommabacillen. Vereinzelte Epithelzellen. Viele rothe } \\
\text { Blutkörperchen. }\end{array}$ \\
\hline-30 Stuuden & $\begin{array}{l}\text { Viele Kommabacillen. Wohl erhaltene Epithelzellen. Keine } \\
\text { rothen Blutkörperchen. }\end{array}$ \\
\hline 18 Stunden & $\begin{array}{l}\text { Vereinzelte Kommabacillen. Reichliche Epithelzellen. Blut } \\
\text { nicht zu erkennen. (Präparat verdorben!. }\end{array}$ \\
\hline über 60 Stunden & $\begin{array}{l}\text { Viele rothe Blutkörperchen. Neben sehr vielen anderen ziem- } \\
\text { lich viele Kommabacillen. Viele wohl erhaltene Epithelien. }++\end{array}$ \\
\hline - 29 Stunden & $\begin{array}{lr}\text { Reichlich Kommabacillen in Schleimflocken. Wohl erhaltene } \\
\text { Epithelzellen. Keine rothen Blutkörperchen. }\end{array}$ \\
\hline 47 Stunden & $\begin{array}{l}\text { Reichlich Kommabacillen. Wenige Epithelzellen. Sehr viele } \\
\text { rothe Blutkörperchen. }\end{array}$ \\
\hline - 41 Stunden & $\begin{array}{l}\text { Wenige Kommabacillen. Keine Epithelzellen. Sehr viele rothe } \\
\text { Blutkörperchen. (Präparat verdorben). }\end{array}$ \\
\hline - 54 Stunden & $\begin{array}{l}\text { Wenige undeutliche Kommabacillen. Sehr viele Epithelzellen. } \\
\text { Vereinzelte rothe Blutkörperchen. (Präparat verdorben). }\end{array}$ \\
\hline-43 Stunden & $\begin{array}{l}\text { Kommabacillen fraglich. Keine Epithelzellen. Vereinzelte rothe } \\
\text { Blutkörperchen. (Präparat verdorben). }\end{array}$ \\
\hline
\end{tabular}




\begin{tabular}{|c|c|c|c|c|c|c|c|}
\hline $\mathrm{Nr}$. & Name & Geschlecht & $\begin{array}{l}\text { Alter } \\
\text { Jahre }\end{array}$ & \multicolumn{2}{|c|}{ Wann erkrankt } & \multicolumn{2}{|c|}{ Wann gestorben } \\
\hline 67 & Golapy (Hindu) & weiblich & 15 & 9. Mai & a. $\mathrm{m}$. & 10. Mai & $5^{\mathrm{h}}$ p. m. \\
\hline 68 & Jeffer (Muhamedaner) & männlich & 60 & 9. , , & a. m. & $9 .$, & $9 \mathrm{~h}$ p. m. \\
\hline 69 & Poymbati (Hindu) & " & 40 & 28. Juni $7^{\text {h }}$ & p.m. & 29. Juni & 5 b a. m. \\
\hline 70 & Kala Nath (Hindu) & - & - & 5. Juli & a. $\mathrm{m}$. & 5. Juli & $8 \mathrm{~h}$ p. m. \\
\hline 71 & Demockamar (Hindu) & männlich & 60 & 7. , & p. m. & 8. , , & $5_{\text {h }}$ p. m. \\
\hline 72 & Gungaram (Hindn) & , & 40 & 18. , , & üh & 19. , , & $8 \mathrm{~h}$ a. $\mathrm{m}$. \\
\hline 73 & Luckli (Hindu) & weiblich & 38 & 29. , $\quad$ Mi & ittags & 30. , , & $11 \mathrm{~h}$ a. $\mathrm{m}$. \\
\hline 74 & Blicku (Hindu) & männlich & 36 & 2. Sept. $8^{h}$ & a. m. & 3. Sept. & $2 \mathrm{~h}$ a. $\mathrm{m}$. \\
\hline 75 & Shile Prasad (Hindu) & , & 16 & 5. , , & p. m. & 6. , & $5^{\text {h }}$ a. m. \\
\hline 76 & Jugudis (Hindu) & " & 24 & 27. Novbr. & a. $\mathrm{m}$. & 29. Nov. & $6^{\mathrm{h}}$ p. m. \\
\hline 77 & Jarangy (Hindu) & , & Jüngl. & 27. , & a. $\mathrm{m}$. & 30. , & $9 \mathrm{~h}$ a. $\mathrm{m}$. \\
\hline 78 & Bipro Dass (Hinda) & , & 56 & 30. , & a. $\mathrm{m}$. & 30. , & $\mathbf{5}^{\mathrm{h}}$ p. m. \\
\hline 79 & Madau (Hindu) & " & 25 & 15. Decembe & & 17. Dec. & $4^{\text {h }}$ p. m. \\
\hline 80 & Behory (Hindu) & ", & 18 & 20. & Früh & 21. , & $2^{b}$ p. m. \\
\hline 81 & Sookari (Hindu) & , & 50 & 28. & ", & $29 .$, & 5 h p. m. \\
\hline 82 & Chatran (Hindu) & ", & 25 & 20. März $12^{\mathrm{h}}$ & a. m. & 22. März & $5^{\text {h }}$ p. m. \\
\hline 83 & $\begin{array}{l}\text { Jaraguddi (Muhame- } \\
\text { daner) }\end{array}$ & " & 25 & 21. $" 9^{\mathrm{h}}$ & a. $m$. & 21. ," & $5^{\mathrm{h}}$ p. m. \\
\hline 84 & Pratai (Hindu) & , & 40 & 25.,$\quad 7^{\mathrm{b}}$ & a. $\mathrm{m}$. & 26. ," & $6 \mathrm{~h}$ a. $\mathrm{m}$. \\
\hline 85 & $\operatorname{Ramn}($ Hindu) & ", & 40 & 26., & a. $\mathrm{m}$. & 26. , , & $8^{\text {h }}$ p. m. \\
\hline 86 & Moula Sing & - & - & 15. April $6 \mathrm{~h}$ & a. m. & 15. April & $6^{\text {h }}$ p. m. \\
\hline 87 & Bhudda (Hindu) & nännlich & 35 & 22. , & a. $\mathrm{m}$. & 22. , , & $5^{\mathrm{h}}$ p. m. \\
\hline 88 & $\begin{array}{l}\text { Rosen Ali (Muhame- } \\
\text { daner) }\end{array}$ & " & 35 & 30. , & a. $\mathrm{m}$. & 30. , & $4^{\mathrm{h}}$ p. m. \\
\hline 89 & Chondroo (Hindu) & ", & 40 & 12. Mai $4^{\mathrm{h}}$ & a. $\mathrm{m}$. & 12. Mai & $5^{h}$ p. m. \\
\hline 90 & Rama Nath (Hindu) & " & 28 & 4. Juni. & & 4. Juni & $6^{\mathrm{h}} \mathrm{p} \cdot \mathrm{m}$. \\
\hline
\end{tabular}




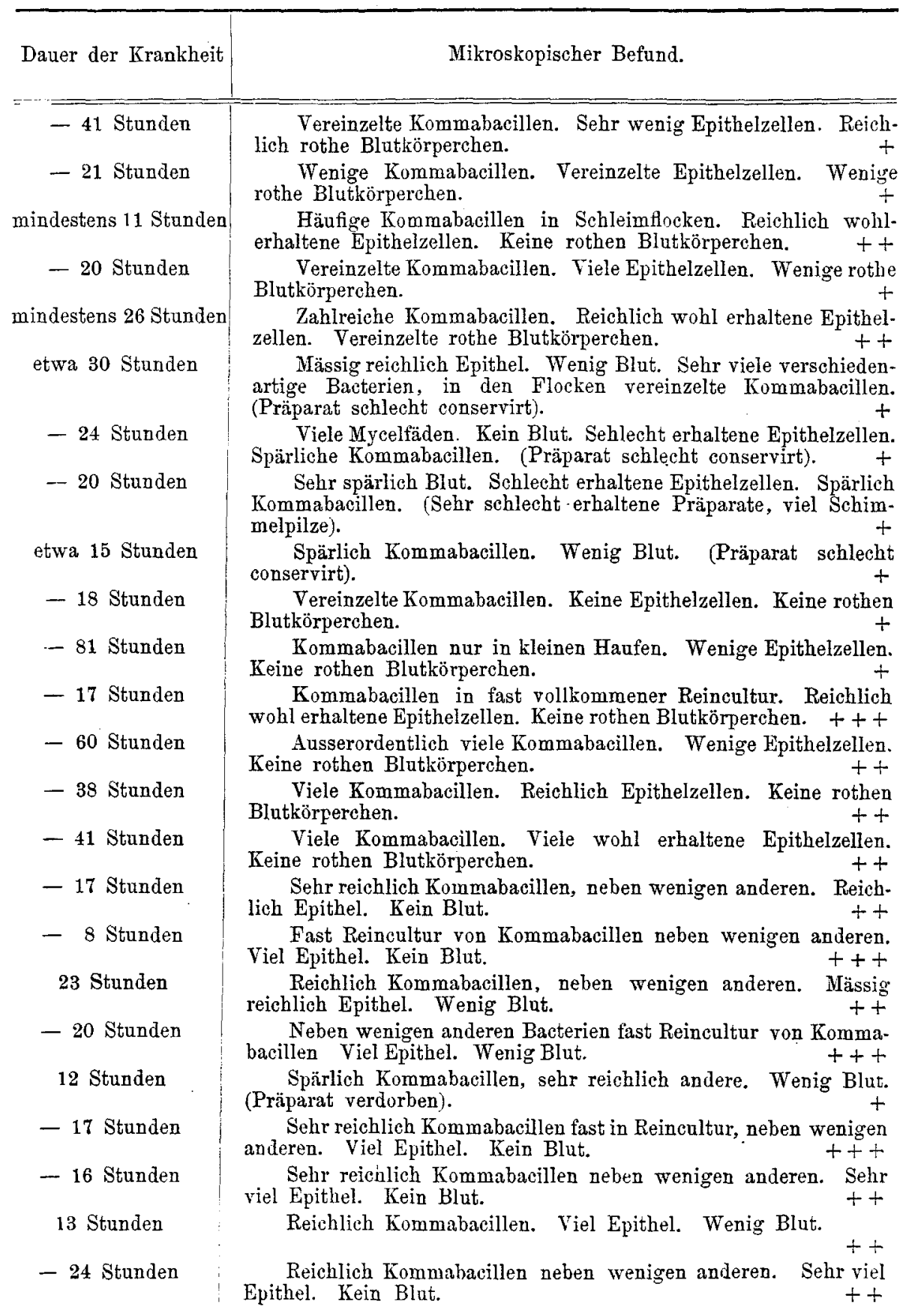


Vergleichen wir weiterhin die in Krankheitsdauer mit der Menge der in jedem Falle constatirten Kommabacillen, so finden wir, dass von 13 Fällen „Reincultur" 12 innerhalb der ersten 24 Stunden, einer innerhalb 30 Stunden gestorben waren; von 32 Fällen ,reichliche Kommabacillen" dauerte die Krankheit bis zu 24 Stunden 13 mal, bis zu 48 $9 \mathrm{mal}$, bis zu $724 \mathrm{mal}$, bis zu $962 \mathrm{mal}$, unbestimmbar war die Krankheitsdauer in 4 Fällen; von den 38 Fällen ,wenige Kommabacillen" dauerte die Krankheit bis zu 24 Stunden $13 \mathrm{mal}$, bis zu $4812 \mathrm{mal}$, bis zu $726 \mathrm{mal}$, bis zu $964 \mathrm{mal}$, bis zu $1201 \mathrm{mal}$, bis zu $1231 \mathrm{mal}$, einer wurde entlassen.

1. Die mikroskopische Untersuchung allein genügt demnach in den meisten Fällen, die Anwesenheit der Kommabacillen im Inhalt des Choleradarmes festzustellen.

2. Auch bei ganz acutem Verlaufe der Cholera werden die Kommabacillen stets im Darminhalte gefunden.

3. Im Allgemeinen ist die Menge der Cholerabacterien eine um so grössere, in je früherem Stadium der Krankheit der Tod eintritt; erfolgt der Tod nach längerer Dauer der Krankheit, so ist die Menge der Cholerabacterien auch eine geringere.

4. Stirbt der Kranke nicht im Choleraanfalle selber, sondern an einer demselben sich anschliessenden Krankheit (Choleratyphoid), so können die specifisehen Cholerabacterien vollständig fehlen.

Am Schlusse unserer Mittheilung möchten wir noch auf die diesbezüglichen Untersuchungen Emmerich's zurückiommen, der auch betreffs dieser Gesichtspunkte $\mathrm{zu}$ wesentlich anderen, ron denen der übrigen Forscher abweichenden Anschauungen gelangt ist.

Zunächst äussert ${ }^{1}$ er sich über den bacteriologisehen Befund bei Choleradejectionen folgendermaassen:

„Auch eine andere, allgemein verbreitete Ansicht ist unrichtig, nämlich die, dass die Koch'schen Vibrionen die einzigen kommaförmigen Pilze der Choleradejectionen seien.

Bis jetzt hat noch Niemand darauf hingewiesen, dass ausser den Koch'schen Kommabacillen noch mehrere andere kommaförmige Vibrionen im Cholerastuhl und im Darminhalte von Choleraleichen häufig vorkommen.

Koch selbst äussert sich nicht über diese diagnostisch vielleicht gleichfalls verwerthbaren Kommabacillen.

1 Untersuchungen über die Pilze der Cholera asiatica. Archiv für Hygiene. Bd. III. Hft. 3 u. 4. S. 295. 
Es giebt überhaupt kaum eine Krankheit, bei welcher in den Dejectionen eine so grosse Menge von sehr verschiedenartigen Vibrionen und Spirillen vorhanden ist, wie in manchen Dejectionen von Cholerakranken.

Stets in vorherrschender Zahl trifft man die auch in den inneren Organen vorhandenen Kurzstäbchen, dann ausserdem mehrere Spirillenformen, kurze sehr dicke, lange und dicke, sehr feine kurze und feine lange Spirillen, flachere und steilere Schrauben, sowie kommaförmige Vibrionen, deren Länge die der Koch'schen Kommabacillen um das Doppelte bis Dreifache übertrifft. Ausserdem finden sich Vibrionen, die den Koch'schen sehr ähnlich sind, aber fein zugespitzte Enden haben."

Zum Beweise aller dieser Behauptungen bringt Emmerich nur eine Zeichnung bei.

„Eingehende, auf zahlreiche Fälle, so fährt Emmerich fort, ausgedehnte Untersuchungen werden vielleicht zeigen, dass ausser den Koch'schen Vibrionen anch noch andere Spaltpilze stete Bewohner des Cholera-Darmes seien."

Emmerich sagt also, dass ausser den Koch'schen Kommabacillen „noch andere kommaförmige Vibrionen" im Cholerastuhle u. s. w. häufig vorkommen.

Nun haben über eine solche Beobachtung die eben angeführten Forscher niemals etwas mitgetheilt; rermuthlich doch nur aus dem Grunde, weil sie von diesen verschiedenartigen Vibrionen und Spirillen nichts zu entdecken im Stande waren. Zudem hat kein einziger Forscher angegeben, dass den Choleraspirillen ähnliche gekrümmte Spirillenformen in wirklich nennenswerther Menge im Darmsecrete bei Cholera auftreten; eine Thatsache, die wir auf Grund unserer zahlreichen Untersuchungen nur bestätigen können. Was aber das auffallendste an diesem ganzen Berichte ist: Emmerich hat offenbar niemals Culturen von diesen „,sehr verschiedenartigen Vibrionen u. s. w." zu Stande gebracht; wenigstens ist nirgends solcher Culturen Erwähnung gethan. Er verfällt also in denselben Fehler, den seiner Zeit Lewis begangen hat, als er nur auf Grund mikroskopischer Untersuchungen den im Mundsehleim vorkommenden gekrümmten Bacillus für identisch mit dem Kommabacillus der Cholera asiatica erklärte. Die Kritik, welche Lewis ron Seiten Koch's zu Theil wurde, und der Tadel, dass eine solche Art der Forschung, welche die Fortschritte der bacteriologisehen Untersuchungsmethoden einfach vernachlässige, unzulässig sei, trifft Emmerich daher in verstärktem Maasse.

Noch grösseres Erstaunen als die oben wiedergegebenen Sätze musste die Behauptung Emmerich's hervorrufen, ,dass auch im normalen Darme die Koch'schen Vibrionen vorkommen" (S. 358 a. a. 0.). 
Diese Behauptung ist geradezu falsch.

Die Thatsache, dass der Koch'sche Kommabacillus ausschliesslich bei Cholera asiatica vorkomme, wird sogar von Buchner, dem enragirtesten Gegner der Koch'schen Lehre von der Aetiologie der Cholera zugegeben mit den Worten: ,die Behauptung Koch's, dass der von ihm aufgefundene Vibrio dem Choleraprocess ausschliesslich eigenthümlich sei, steht bis jetzt unerschüttert fest (S. 438 a. a. 0.).“ Jedenfalls wird man die in einer Bemerkung zu obiger Behauptung von Emmerich hinzugefügte Angabe, dass die „Genese" von Kommabacillen im Choleradarme durch die Beobachtung des Vorkommens von zierlichen starkgekrümmten Vibrionen in dem mucinreichen Kothe der Wald- und Weinbergschnecke in gewisser Weise beleuchtet werde, nicht als Beweis ansehen können. Ganz abgesehen davon, dass hier wieder die Kommabacillen der Cholera ohne weiteres in Beziehung zu den Vibrionen des Schneckenkothes gebracht werden, dürften für die „Genese" von Kommabacillen die fäulnisswidrigen Eigenschaften des mucinreichen Transsudates in keiner Weise eine Erklärung zu liefern im Stande sein, höchstens doch nur für eine ungehinderte fernere „Entwickelung" der in dieser ihnen zusagenden Nährflüssigkeit vorhandenen gekrümmten Bacillen.

Noch eine weitere Angabe von Emmerich bedarf der Erörterung.

Am Schlusse seiner ersten Mittheilung ${ }^{1}$ schreibt er wörtlich Folgendes:

„Auf den Darmbefund bei menschlicher Cholera wirft die Beobachtung einiges Licht, dass nach Injection der (Neapler) Cholerabacterien unter die Haut oder in die Lungen die 10 oder 15 normal im Meerschweinchendarme vorkommenden Pilze verschwinden, während in den pathologischen Darmsecreten kommaförmige Bacillen und Spirillen auftauchen, schliesslich aber nur einige Arten übrig bleiben."

Der Sinn dieses Satzes ist nicht ganz leicht zu verstehen.

„Die Spaltpilze des normalen Meerschweinchendarmes rerschwinden; dafür treten (anfänglich?) kommaförmige Bacillen und Spirillen (in grösserer Anzahl?) auf, schliesslich bleiben nur einige Arten ron kommaförmigen Bacillen und Spirillen übrig."

Die eingeklammerten Worte ergeben sich aus dem Sinne des Citates und sind ron uns hinzugefügt, um die Widersprüche die in dem so gefassten Satze enthalten sind, noch deutlicher zu machen.

Leider hat Emmerich in der ausführlichen Arbeit dieser Beobachtung kein weiteres Wort gewidmet, was sie wohl verdient hätte, so dass wir uns an den obigen kurzen Satz halten müssen.

${ }^{1}$ Ueber die Cholera in Neapel u. s. w. Archiv für Hygiene. Bd. II. S. 422. 
Wann hat Emmerich dieses Auftauchen ron kommaförmigen Bacillen u. s. w. beobachtet? Hat er von seinen Versuchsthieren einige vorzeitig getödtet, um diese Beobachtung zu machen?

„Schliesslich", das soll doch wohl heissen, nach dem Tode des Thieres bleiben nur einige Arten übrig? Wie hat er constatirt, dass diese angeblich auftretenden kommaförmigen Bacillen verschiedene Arten sind? Nur durch die milkroskopische Untersuchung? Und wie stellt sich Emmerich diesen ganzen Vorgang vor? Soll dieser Satz etwa so zu rerstehen sein, dass die Neapler Bacterien, die ja bekanntlich Stäbchen sind, bei ihrem Uebergange in den Darm sich komma- oder spirillenfürmig krümmen und verändern? Oder aber verändern sich von den 10-10 normal im Meerschweinchendarme vorkommenden Spaltpilzarten einige in Komma's und Spirillen? Eine andere Möglichkeit der Genese ist doch nicht vorhanden. Wie aber auch Emmerich sich aussprechen mag; mit dieser Behauptung stellt er alles, was die bacteriologische Forschung in den letzten Jahren an sicheren Thatsachen über die Constanz der Arten der Bacterien beigebracht, in Abrede und öffnet der ungezügelten Speculation auf diesem Gebiete wieder Thür und Thor, welche Dank der sorgsamen, vorsichtig in ihren Schlüssen sich bewegenden Arbeiten der ätiologischen Forschung der Infectionskrankheiten für immer verbannt und ausgesehlossen schien. 